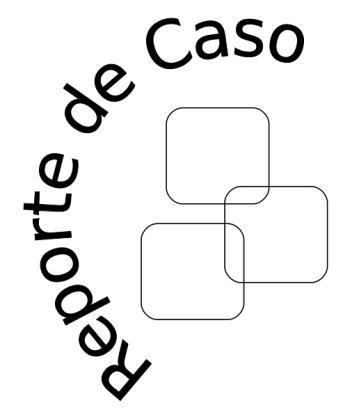

César Augusto Solano Villanueva Universidad Distrital Francisco José de

Caldas.

casolanov@correo.udistrital.edu.co

Johan Fabiel Casas Díaz

Universidad Distrital Francisco José de

Caldas.

jfcasasd@correo.udistrital.edu.co

Juan Carlos Guevara Bolaños

Universidad Distrital Francisco José de

Caldas.

jcguevarab@udistrital.edu.co

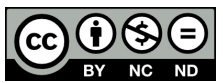

Citación: Solano, C. A., Casas J. F. y Guevara, J. C. (2015). Aplicación móvil de realidad aumentada para la enseñanza de la clasificación de los seres vivos a niños de tercer grado. En: Ingeniería, Vol. 20, No. 1, pp. 79-93

\section{Aplicación móvil de realidad aumentada para la enseñanza de la clasificación de los seres vivos a niños de tercer grado}

\section{A Mobile Augmented Reality Application for Teaching the Classification of Living Things to Primary School's Children}

\section{Resumen}

Las tecnologías de la información y las comunicaciones (TIC) han tenido un gran impacto en los últimos años en el aula de clase, obteniendo resultados prometedores en el apoyo al proceso educativo. Lo anterior hace indispensable crear herramientas innovadoras que fortalezcan los procesos de enseñanza y aprendizaje de los estudiantes, además de motivarlos a usar estas tecnologías tanto fuera como dentro del aula. En este artículo se presenta el desarrollo de una herramienta de este tipo, en donde se incorporan mecanismos de Realidad Aumentada (RA) en la enseñanza de temas biológicos en niños de tercer grado de primaria, y adicionalmente se plantea el modelo de evaluación que se utilizará para medir el impacto del software.

Palabras claves: ciencias naturales, constructivismo, educación primaria, objetos virtuales, realidad aumentada.

\section{Abstract}

The Information and Communication Technologies (ICT) have had an important development in recent years with promising results in supporting learning processes in the classroom . For this reason, it is important to create innovative tools to motivate teachers and students to use these kind of technologies both inside and outside the classroom. This paper describes the development of a tool in this direction, aimed at helping students of third grade of primary school in learning classification of living beings aided with Augmented Reality (AR) techniques. Additionally an evaluation model is proposed to measure the impact of the software.

Key words: augmented reality, constructionism, natural science, primary education, virtual objects. 


\section{Introducción}

Las tecnologías de la información y las comunicaciones (TIC) son herramientas tecnológicas que permiten acceder, producir, guardar, presentar y transferir información [1]; dichas herramientas se han abierto paso a través de los años permeando cada uno de los aspectos de nuestra vida (social, político, económico, cultural, etc.) con lo que han demostrado el gran impacto que poseen. Sin embargo, existe una brecha acentuada en los países en vías de desarrollo conocida como la brecha digital. Entre los esfuerzos por disminuir dicha brecha, el factor económico adquiere una gran importancia, aunque en algunos casos la educación también juega un papel importante. Es por esto que la formación en TIC de los docentes y el respectivo acercamiento a sus estudiantes es un factor sustancial en la eliminación de la brecha digital [2].

Numerosos estudios han demostrado que en todo proceso de cambio o renovación de los procesos de enseñanza-aprendizaje de las ciencias, los docentes son el componente clave, en razón a que deben estar convencidos de que se necesita de su innovación, creación y actitud hacia el cambio para responder a los planteamientos y propósitos que se fijan en las propuestas didácticas, así como para cumplir con las exigencias de los contextos [3]. Sin ellos, adoptar nuevos modelos o modificar los ya existentes sería imposible.

La ciencia y la tecnología (CyT) tienen una presencia ubicua, notoria y significativa en la sociedad. Sus repercusiones en la economía, la política, la educación, la cultura, el ocio, etc., ponen de relieve su destacado papel en las decisiones, tanto personales como colectivas [4]. En una sociedad globalizada como la actual se observa un alto grado de consenso en cuanto a la necesidad de practicar una enseñanza científica capaz de formar alumnos como ciudadanos, en el sentido de que puedan usar la información científica en el momento de tomar decisiones de forma fundamentada. Para conseguir esto en la educación primaria, se debe plantear una ciencia que enseñe a pensar, hacer, hablar, a regular los propios aprendizajes y trabajar en interacción [5]. Se trata de acercar la ciencia a los intereses de los alumnos abordando las implicaciones sociales y éticas que el impacto tecnológico conlleva; este enfoque facilitará el uso en la vida diaria de lo aprendido en la escuela. Bajo este prisma, la enseñanza de las ciencias deja de concebirse como una opción para alumnos de élite y se convierte en un instrumento para la alfabetización tecnológica de los ciudadanos que los ayuda a comprender los problemas que tiene la sociedad actual y los faculta para la toma de decisiones fundamentadas y responsables [6].

Ahora bien, se han identificado varios problemas de la enseñanza de las ciencias a nivel mundial: el primero es la gran cantidad de profesores que no tienen la formación adecuada, lo cual se puede traducir en una falta de confianza en su capacidad de enseñar ciencias adecuadamente. Segundo, en la mayoría de los currículos de las distintas ciencias se abarcan demasiados temas y contenidos, lo cual no solo hace más difícil la labor docente, sino que además interfiere con la posibilidad de que el estudiante haga el ejercicio necesario de reflexión, lo cual es fundamental para la compresión de cualquier tema [7]. Y por último, se puede apreciar que los científicos han dado respuesta a los diferentes fenómenos, pero este conocimiento queda plasmado en los textos que ellos mismos elaboran, con un lenguaje bastante avanzado, y estas respuestas deben ser conocidas por todas las generaciones, las cuales deben tener acceso al conocimiento científico. 
La enseñanza de las ciencias en Colombia ha seguido un desarrollo paralelo al que han tenido los correspondientes estudios en el mundo. Sin embargo, ha presentado problemas como: el aprendizaje memorístico y repetitivo que no lleva a una comprensión real de las teorías y las explicaciones, la permanencia de ideas previas, preconceptos o preteorías que se oponen a las explicaciones y teorías de las ciencias, la incapacidad por parte de los estudiantes de resolver verdaderos problemas, el desconocimiento por parte de los maestros de los modos de conocimiento de los estudiantes, el temor de los estudiantes de expresar el propio punto de vista y discutirlo y defenderlo, la ausencia de autocrítica y de flexibilidad [8].

Con el fin de delimitar el problema, se eligió el tema de la clasificación de los seres vivos en el grado de tercero de primaria, ya que es un concepto estructurante de la biología, además de que se imparte en varios años de escolaridad, aunque es en el grado tercero donde se inicia este proceso [9].

Debido a estos problemas en la educación, el Ministerio de Tecnologías de la Información y las Comunicaciones de Colombia creó la iniciativa “Tabletas para educar", liderada por Computadores para Educar, mediante la cual se beneficia a las instituciones educativas públicas del país con tabletas con el propósito de fortalecer las dinámicas de aprendizaje de los estudiantes, favorecer la inclusión social y generar más y mejores oportunidades en cada región de la mano de la tecnología. Las tabletas son herramientas óptimas para la distribución, producción y consumo de contenidos educativos de distintos formatos y características: leer, jugar, ver videos, editar, producir estructuras, etc. [10]. Por esto se hace evidente la necesidad de crear aplicaciones para dispositivos móviles que estén orientadas y diseñadas con fines educativos y permitan mejorar los procesos educativos de las instituciones favorecidas por este programa.

La idea de usar software educativo de realidad aumentada (RA) para facilitar los procesos de enseñanza en las aulas de clase va ligada estrechamente al modelo constructivista, el cual se basa fundamentalmente en dos principios: el primero indica que el conocimiento no es recibido pasivamente, sino construido activamente por el sujeto pensante que realiza el acto del conocimiento; el segundo establece que la función de la cognición es adaptativa y sirve a la organización del mundo experiencial, no al descubrimiento de una realidad ontológica [11]. Estos principios permiten usar las TIC como herramientas del constructivismo para incentivar al estudiante a ser parte activa en la construcción de su propio conocimiento.

Desde su creación, la RA ha sido motivo de interés para diversos investigadores quienes vieron en esta tecnología un gran potencial para diversos campos de las ciencias naturales, entre ellas la clasificación de los seres vivos; además, su uso en la educación permite que los estudiantes difuminen en cierta medida las fronteras del mundo real y el digital.

Debido a las ventajas que proporciona el uso de RA en el área de la educación, se ha planteado realizar una aplicación que permita enseñar las clasificación de los seres vivos, la cual además de permitir visualizar contenido virtual, permitirá a los estudiantes enlazar sus experiencias con los conceptos aprendidos en el aula de clase con el fin de que puedan realizar un reflexión de lo aprendido. Por su parte, el docente tendrá la posibilidad de monitorear el uso y avance de la aplicación para que pueda medir y retroalimentar sus estrategias educativas. 
El presente artículo se divide en las siguientes secciones: revisión de la literatura, donde se describe qué es la RA y cuáles han sido las aplicaciones más significativas que han surgido para esta tecnología; luego, en metodología, se indican cuáles metodologías usar y la forma en que estas se combinan para alcanzar nuestro objetivo; en herramientas de desarrollo se hace una breve descripción las tecnologías más importantes para llevar a cabo este proyecto; en descripción de la solución se relata cómo se integran los diferentes componentes del proyecto para crear nuestra aplicación y se describen todas las funcionalidades del sistema; en la sección de resultados se presentan los avances alcanzados en la aplicación y el modelo de evaluación tecnológica que se usará; para finalizar se encuentran las conclusiones obtenidas hasta el momento, el trabajo futuro en el cual se plasman algunas ideas acerca de posibles proyectos que se puedan derivar a partir de este trabajo y las referencias utilizadas en el texto.

\section{Revisión de la literatura}

Los sistemas de RA son sistemas que combinan el mundo real y el virtual para ayudar al usuario a realizar una tarea en el mundo físico. Esta tecnología ha despertado el interés de muchos investigadores debido a que existe una gran necesidad por parte de los usuarios en beneficiarse tanto de las computadoras como del mundo real. La RA incorpora contenidos de realidad virtual (RV), pero se diferencia de esta, pues la RV se encuentra aislada del mundo y sus escenarios son puramente virtuales, mientras los sistemas de RA presentan las siguientes características [12]:

- Agregan información gráfica en 3D al mundo real

- Agregan información de audio dentro del mundo real

- Usan un feedback muy fuerte como posible método para aumentar las capacidades del usuario para interactuar con el mundo real.

Una vez la RA es llevada al aula de clase para el uso en la enseñanza de las ciencias, se pueden apreciar las siguientes ventajas [7]:

- Aumenta y apresura la capacidad de trabajo de los alumnos

- Provee acceso a fenómenos que serían muy difíciles o imposibles de observar de otra forma en una escuela primaria normal

- Ayuda a la experimentación porque proporciona una retroalimentación visual inmediata

- Enfoca la atención de los alumnos en aspectos poco obvios y resalta así conceptos abstractos

- Propicia el aprendizaje colaborativo y autorregulado

- Aumenta la motivación y el compromiso de los alumnos. 
A continuación se presentan algunas de las aplicaciones de realidad aumentada que han incursionado en la educación y que han obtenido mayor popularidad.

Anatomy 4D: es una aplicación para dispositivos móviles IOS y Android. Su objetivo es permitir a los estudiantes de todo el mundo aprender la anatomía de forma rápida y precisa, en cualquier lugar y a cualquier hora. Su diseño permite que la puedan utilizar desde estudiantes de colegio hasta profesionales de la medicina. Esta aplicación fue desarrollada con el SDK de Vuforia en conjunto con Unity3d, en la Figura 1 se puede apreciar un ejemplo de la aplicación.

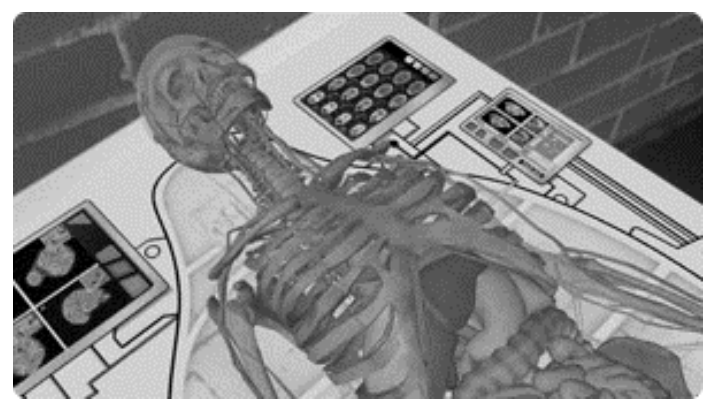

Figura 1. Ejemplo Anatomy 4D.

Fuente: [13]

Sesame Street: permite a los niños usar un teléfono inteligente como un ojo digital en ambientes reales para encontrar, identificar y aprender acerca de las palabras de su entorno, con lo que ayuda a una mayor comprensión de su significado mediante la exposición de los niños a ellas en contextos familiares. En la Figura 2 se puede ver una imagen de esta aplicación.

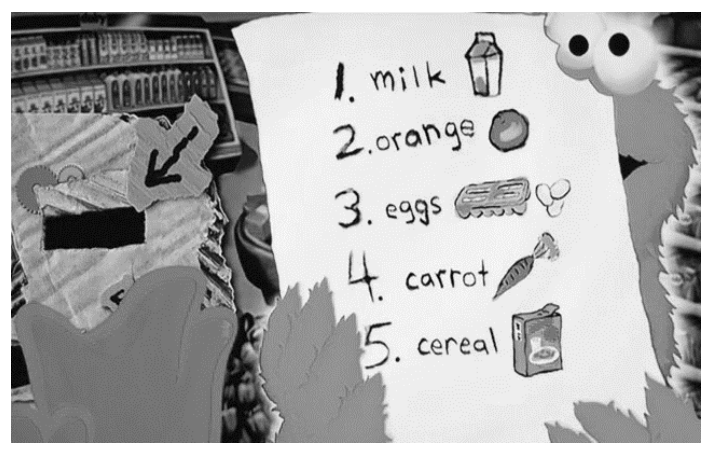

Figura 2. Imagen ilustrativa de la aplicación Sesame Street.

Fuente: [14]

Por último está la revista médica Neurosurgery, revista oficial del Congreso de Cirujanos Neurológicos, que publica más de 3.000 páginas de contenido cada año y utiliza la realidad aumentada para dar al lector una mejor compresión de los artículos publicados en la revista.

La RA puede ser considerada como una de las muchas formas de software educativo, el cual se define como programas de computación realizados con el objetivo de ser empleados en la mejora de los procesos de enseñanza y, consecuentemente, de aprendizaje. Existen varias clasificaciones de software educativo: por tipo de aplicación, por su función educativa 
o por su fundamentación educativa, pero independientemente de esto, deben poseer ciertas características para que pueda ser considerado educativo (Tabla I) [15].

Tabla I. Características del software educativo

\begin{tabular}{|l|l|}
\hline \multicolumn{1}{|c|}{ Características } & \multicolumn{1}{c|}{ Descripción } \\
\hline Facilidad de uso & $\begin{array}{l}\text { Deben ser en lo posible autoexplicativos y con sistemas } \\
\text { de ayuda }\end{array}$ \\
\hline Capacidad de motivación & Deben mantener el interés de los alumnos \\
\hline Relevancia curricular & Deben estar relacionados con las necesidades del docente \\
\hline Versatilidad & Deben ser adaptables al recurso informático disponible \\
\hline Enfoque pedagógico & Den ser actuales: constructivista o cognitivista \\
\hline Orientación hacia los alumnos & Deben tener control del contenido del aprendizaje \\
\hline Evaluación & Deben incluir módulos de evaluación y seguimiento \\
\hline
\end{tabular}

\section{Metodología}

Dado el carácter multidisciplinar del proyecto, fue necesario analizar varias metodologías que permitieran establecer una guía con respecto a cada uno de los componentes conceptuales que lo integran, lo cual dio como resultado el uso de tres metodologías: la primera es Scrum, usada para el desarrollo del software de una manera ágil y rápida [16]; la segundo es AODDEI (Análisis, Obtención, Diseño, Desarrollo, Evaluación, Implementación) para el desarrollo de la parte pedagógica [17]; y por último está el modelo TAM (Technology Acceptance Model), que predice cómo los usuarios aceptan y utilizan una herramienta tecnológica [18]. En la Tabla II se describe cómo se integran estas metodologías.

Tabla II. Integración de metodologías para el desarrollo del proyecto

\begin{tabular}{|l|l|l|c|}
\hline \multicolumn{1}{|c|}{ Metodología Fase } & \multicolumn{1}{c|}{ Scrum } & \multicolumn{1}{c|}{ AODDEI } & \multicolumn{1}{c|}{ TAM } \\
\hline Requerimientos & Product Backlog & - & $\begin{array}{l}\text { Definición de las } \\
\text { variables }\end{array}$ \\
\hline Análisis & $\begin{array}{l}\text { Spring Planning } \\
\text { Meeting }\end{array}$ & $\begin{array}{l}\text { Análisis y obtención } \\
\text { del material }\end{array}$ & - \\
\hline Diseño & Sprint Backlog & Armado o creación & - \\
\hline Implementación & $\begin{array}{l}\text { Spring Review, } \\
\text { Sprint Retrospective }\end{array}$ & $\begin{array}{l}\text { Evaluación e } \\
\text { Implementación }\end{array}$ & $\begin{array}{l}\text { Validación del } \\
\text { modelo }\end{array}$ \\
\hline Pruebas & & & \\
\hline
\end{tabular}


Como se puede apreciar, las tres metodologías se descompusieron en cinco fases, que se describen a continuación con las actividades necesarias para el desarrollo del proyecto:

Requerimientos: para el desarrollo del software se establece el Product Backlog, el cual contiene todas las tareas necesarias. En cuanto a TAM, es necesario definir las variables que se utilizarán para la validación del modelo

Análisis y diseño: esta fase es compartida tanto por Scrum como por AODDEI; lo que se hace es planificar las tareas que se realizan en las iteraciones definidas en Scrum, así como la elección del material didáctico educativo para la aplicación de RA.

Implementación: se realizan las tareas definidas en el Sprint Backlog de la aplicación de RA, así como la elaboración del material didáctico que no se haya podido obtener; al finalizar cada iteración se hará el Sprint Review para revisión del producto.

Pruebas: se realizan las pruebas de la aplicación antes de realizar el Sprint Retrospective, una vez finalizado el software, al mismo tiempo que se hará la validación de la tecnología con el modelo TAM.

\section{Descripción de la solución}

La aplicación se utilizará como una herramienta para ofrecer una perspectiva más realista de algunos contenidos como talleres del libro de ciencias naturales elegido; de esta forma, el estudiante podrá percibir más información de un contenido tridimensional digital que en un escenario real, y a su vez podrá resolver con mayor facilidad los talleres planteados. En la Figura 3 se puede ver el modelo conceptual de la aplicación, con cada uno de los módulos que van a ser creados o están en desarrollo, seguido de la descripción y funcionamiento.
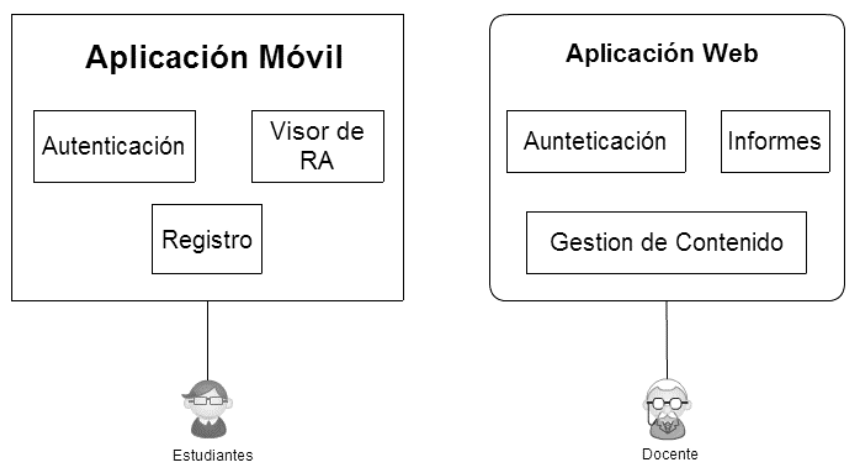

Figura 3. Modelo conceptual de la solución propuesta.

Fuente: los autores

En primer lugar se encuentra la aplicación móvil desarrollada en Android, la cual será de uso enteramente para los estudiantes y poseerá dos módulos: el primero será de autenticación 
y registro de usuarios, el segundo será el de realidad aumentada, encargado de mostrar los modelos en 3D y demás contenidos multimedia en la pantalla del dispositivo. Esto es posible gracias a que el SDK Vuforia permite capturar una imagen e identificar si hay algún elemento utilizado como marcador; si el contenido es un modelo en 3D, se visualizará inmediatamente, puesto que ya se encuentra almacenado en el dispositivo para dar un mejor rendimiento. En caso de que sea cualquier otro contenido multimedia, se establecerá una comunicación hacia los servicios web por medio de JavaScript con el fin de recuperar del servidor dichos recursos y presentarlos al usuario, en este caso el estudiante.

Estos servicios web además de obtener los contenidos multimedia del servidor, también serán los encargados de consultar y almacenar la información del sistema en general a través de una conexión directa a la base de datos, lo que permitirá que en un futuro se pueda interactuar con otras aplicaciones, de ser necesario. Dichos servicios web están creados bajo la arquitectura Representational State Transfer (REST) para dar una mayor flexibilidad y escalabilidad.

Por otro lado, se encuentra la aplicación web destinada a los docentes; su principal objetivo es servirle al profesor como fuente primaria de información acerca del manejo de la aplicación móvil por parte de sus estudiantes, puesto que le permitirá consultar indicadores de uso y avance. Otra de sus funcionalidades es la gestión de los contenidos, es decir, los docentes pueden agregar nuevos contenidos que consideren útiles, como videos, imágenes o audios.

La aplicación web se encuentra dentro del servidor Glassfish junto con los servicios web, y al igual que estos también tendrá una conexión a la base de datos del sistema para que el docente pueda consultar la información de sus estudiantes.

En la Figura 4 se puede apreciar la arquitectura de la aplicación, donde se visualiza más claramente la comunicación entre módulos y tecnologías.

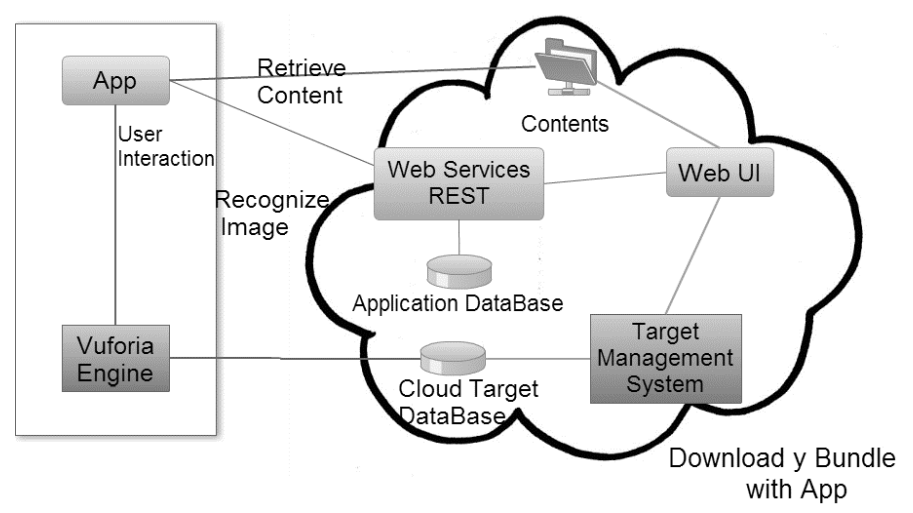

Figura 4. Arquitectura planteada para la aplicación.

Fuente: [19] modificado por los autores 
Por último se describen las tecnologías usadas para el desarrollo de este proyecto, las cuales son:

Lenguaje de Programación: son tres, el primero es Java EE 6 para la aplicación web que mejora significativamente la productividad del desarrollador, presenta un perfil web ligero para aplicaciones web e incluye las últimas versiones de tecnologías como JAX-RS 1.1, JavaServer Faces(JSF) 2.0, Enterprise JavaBeans (EJB) 3.1, Java Persistence (App) 2.0, Context and Dependency Injection (CDI) 1.0 y mucho más. El segundo es Android, ya que es de código abierto y permite llegar a una mayor cantidad de dispositivos. Y por último, JavaScript, el cual es un lenguaje de programación interpretado muy poderoso y popular para la web y que permite mejoras en la interfaz de usuario y páginas web dinámicas.

Servidor de aplicaciones: GlassFish es un servidor de aplicaciones de código abierto compatible con Java EE, listo para funcionar en entornos de producción. GlassFish v3 proporciona una pequeña base con todas las funciones para la implementación de Java EE 6.

Motor de RA: Vuforia es un software developer kit (SDK) para el desarrollo de aplicaciones de RA que usa la pantalla de los dispositivos móviles como lente "mágico" para hacer coexistir el mundo virtual con el mundo real. Permite el reconocimiento de imágenes y objetos del mundo real de una manera fácil y sencilla.

Game Engine: Unity 5 es una plataforma de desarrollo poderosa y flexible para la creación de juegos y experiencias interactivas en $2 \mathrm{D}$ y $3 \mathrm{D}$. Unity es similar a motores de juegos como Blender, Virtools y Torque Game Builder, pero se destaca por su robustez y sencillez.

Base de datos: PostgreSQL es un sistema de gestión de bases de datos objeto-relacional, distribuido bajo licencia BSD y con su código fuente disponible libremente. Es el sistema de gestión de bases de datos de código abierto más potente del mercado. Utiliza un modelo cliente/servidor y usa multiprocesos en vez de multihilos para garantizar la estabilidad del sistema.

\section{Resultados}

Debido a que el presente proyecto aún se encuentra en fase de desarrollo, este artículo pretende presentar los avances alcanzados hasta la fecha con respecto de la aplicación móvil de RA y el modelo de evaluación que se utilizará para medir el impacto del software.

\subsection{Aplicación móvil}

Los resultados obtenidos hasta el momento con respecto a la aplicación móvil de realidad aumentada consisten en el módulo de registro y autenticación (Figura 5). 

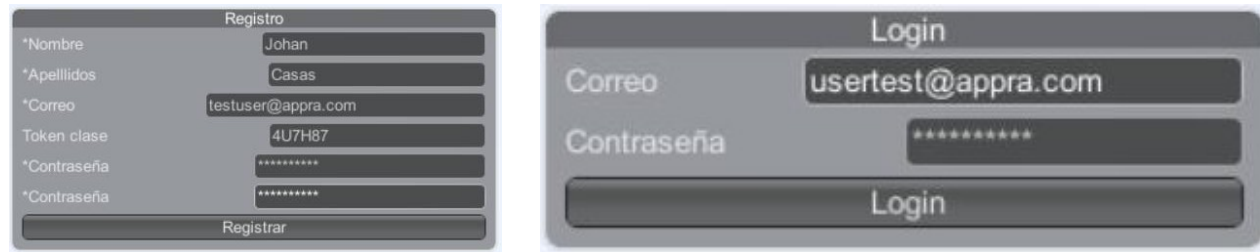

Figura 5. Capturas de pantalla del módulo de registro y autenticación.

Fuente: los autores

En la Figura 6 se aprecia la interfaz principal de la aplicación en la cual se encuentra un menú de opciones, además se habilita la cámara para que el usuario pueda escanear un marcador cualquiera.

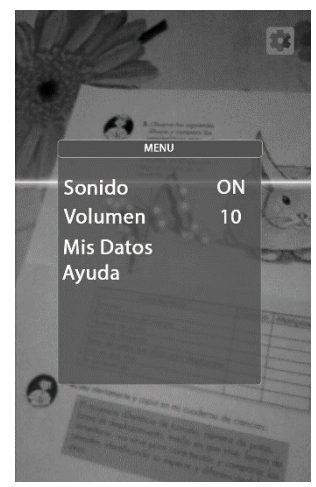

Figura 6. Captura de pantalla de la interfaz principal.

Fuente: los autores

Adicionalmente también se implementó el sistema que escanea los marcadores del libro guía y muestra el respectivo contenido. En la Figura 7 se puede ver el modelo en 3D de una ballena.
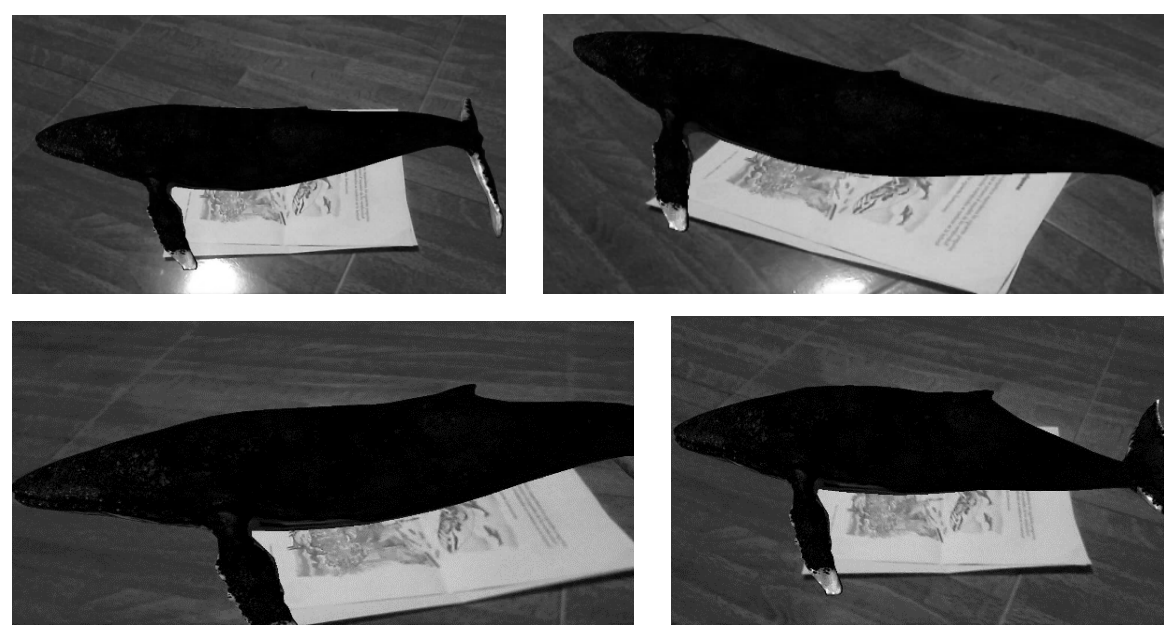

Figura 7. Modelo en 3D de una ballena.

Fuente: los autores 
En la Figura 8 se puede apreciar otro de los modelos en 3D, que corresponde a una mariposa agitando las alas.
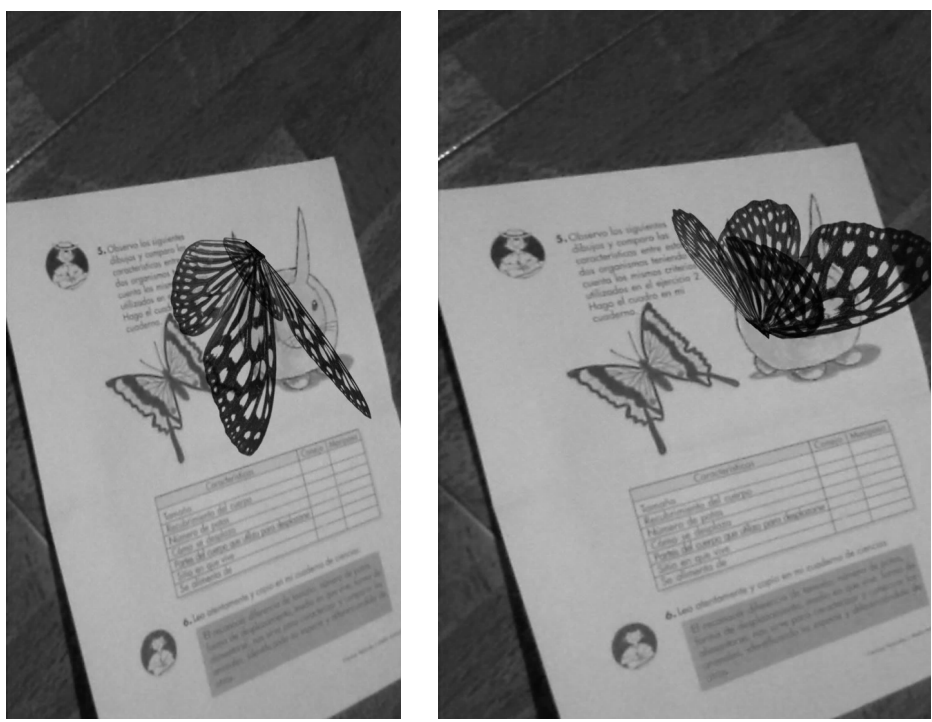

Figura 8. Modelo en 3D de una mariposa.

Fuente: los autores

Por último, en la Figura 9 se presenta el módulo de datos personales de la aplicación web donde el docente puede consultar y modificar sus datos así como el de sus estudiantes.

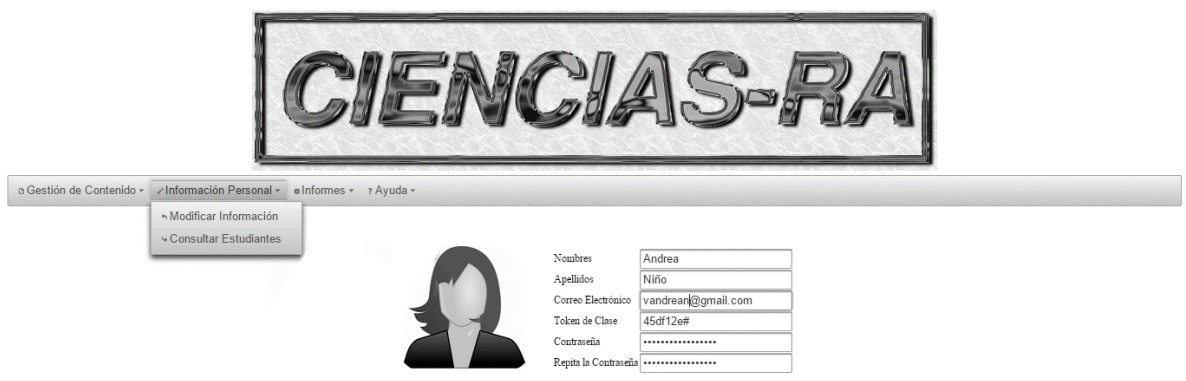

Figura 9. Captura de pantalla de la aplicación web.

Fuente: los autores

\subsection{Modelo de evaluación}

Para medir el impacto de la aplicación tanto en estudiantes como docentes se planteó usar una extensión al modelo TAM [18], para ello se aplicarán encuestas que midan la percepción del usuario con respecto a dos tipos de variables (dependientes e independientes). 
Dentro de este modelo extendido se encuentran cuatro variables independientes: la primera es la utilidad estimada (EU), la cual deberá ser establecida por evaluadores externos; la segunda es la facilidad de uso percibida (PEOU), que indica el grado en que una persona cree que podrá usar un sistema sin realizar ningún esfuerzo; la tercera es la intención hacia el uso (BI), que consiste en el grado en el que una persona ha formulado planes conscientes para desarrollar (o no) alguna conducta futura; y la cuarta es el uso (U).

Por otro lado, las variables independientes son tres, las cuales permiten determinar la facilidad de uso de percibida: la primera es la afectividad frente al sistema, la segunda es autoeficacia con respecto al sistema y la tercera es la experiencia usando el sistema.

Como ya se mencionó, estas variables serán medidas a través de la aplicación de encuestas según el modelo extendido de TAM, el cual indica qué aspectos se deben tener en cuenta al momento de formular las preguntas que evalúan cada variable, y así mismo señala que se debe hacer una validación de la encuesta antes de aplicarla a los usuarios reales con el fin de obtener feedback. En la Figura 10 se ilustra el modelo de evaluación:

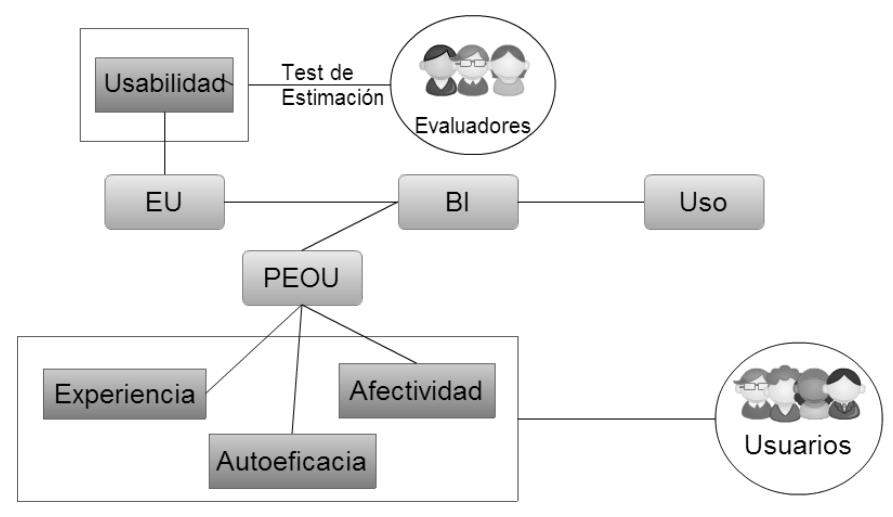

Figura 10. Modelo de evaluación.

Fuente: los autores

Este modelo de evaluación será aplicado a los estudiantes utilizando la estrategia de grupos de control y grupo experimental, donde al primer grupo se le impartirán los temas de clase como se realiza normalmente, mientras que al segundo grupo se le dará acceso a la herramienta y a los cuestionarios que se elaborarán para definir el porcentaje de éxito de la aplicación.

Puesto que este proyecto aún se encuentra en fase de desarrollo, los esfuerzos a los que nos encaminaremos en los próximos meses serán a la culminación exitosa de software y a la realización de las respectivas pruebas bajo el modelo de evaluación explicado. Una vez se hayan culminado estas fases, se procederá a realizar el proceso de feedback o retroalimentación con el fin de implementar este proyecto en una escala mayor. 


\section{Conclusiones}

Debido a que la aplicación aún se encuentra en etapa de desarrollo, no es posible tener resultados acerca del uso e impacto del software en la población que se seleccionará, sin embargo se presentan algunas observaciones que nos parecen útiles con el fin de guiar a otros investigadores que trabajen en temas o tecnologías similares.

Al momento construir los servicios web nos enfrentamos a dos alternativas, utilizar SOAP o REST. Las dos tecnologías poseen sus ventajas, pero se decidió implementar servicios web basados en REST por su sencillez a la hora al momento de implementarlos, además de que permite utilizar JSON, una forma de comunicación más rápida que XML.

En una etapa inicial del proyecto no se contempló la idea de usar JavaScript, pero a medida que el desarrollo fue avanzado, se encontró que la invocación de los servicios web a través de la aplicación móvil realizada sobre Android era bastante compleja. Utilizando JavaScript pudimos simplificar este proceso.

Durante el desarrollo de la aplicación web se encontró que para poder crear un módulo que permita gestionar los contenidos multimedia, el docente debería estar en capacidad de crear nuevos marcadores, para lo cual necesitaría previa capacitación en el uso de determinadas herramientas de desarrollo, lo cual no es objetivo de este proyecto, por consiguiente se debe replantear la forma en que los docentes puedan agregar nuevo material.

La utilización de TAM como modelo de aceptación nos permitirá predecir cómo será la utilización de este software por parte de los usuarios, además, el hecho de utilizar una versión modificada nos permite simplificar el modelo, y reducir tiempo y costos en el momento de obtener los resultados de investigación definitivos.

Por último, se pudo apreciar que en los últimos años se ha producido un gran avance con respecto a la inclusión de nuevas tecnologías en el aula de clase con el fin de apoyar los procesos de enseñanza-aprendizaje, así como un cambio en las actitudes y aptitudes de los estudiantes frente a cómo reciben sus clases, por lo que es necesario que los procesos educativos en la escuela se adapten a estos cambios con el fin de incentivar en el alumno el interés por aprender. Tales cambios deben ser impulsados por los docentes, puesto que estudios previos han demostrado que son ellos quienes por medio de las nuevas herramientas mejoran la experiencia educativa de sus alumnos.

\section{Trabajo futuro}

Una vez este proyecto quede concluido, se puede pensar en hacer una implementación o adaptación a otros dispositivos más allá de los smartphones o tabletas, por ejemplo, en tecnologías emergentes como Google Glass, HoloLens de Microsoft, o incluso llegar a plantear proyectos con realidad virtual utilizando el dispositivo Oculus Rift, donde podríamos simular perfectamente un laboratorio completo de física o química, a muy bajos costos, lo cual es muy 
posible con la arquitectura que se plantea actualmente. Este proyecto es solo una muestra del potencial que poseen estas tecnologías, la punta del iceberg de lo que se podría lograr

\section{Referencias}

[1] Ministerio de Tecnologías de la Información y las Comunicaciones, “En Tic Confio,” Ministerio de las TIC, disponible en: http://www.enticconfio.gov.co/index.php/usosincreibles/39.html.

[2] A. Silva, J. C. Ponce, F. Álvarez, J. Muñoz y C. Collazos, “Red iberoamerica para disminucion de la brecha digital de población vulnerable a través de estrategias de contenidos multiculturales (REDUCE)," LACLO, Volumen 4, Número 1, 2013, pp. 1-10.

[3] A. Henao y L. González, "Elaboración de un ambiente virtual colaborativo usando eXe Learning para la enseñanza de ciencias naturales," Ventana Informática, Número 31, 2014, pp. 115-130.

[4] Á. Vázquez y M.. Manassero, “La selección de contenidos para enseñar naturaleza de la ciencia y tecnología (parte 1): Una revisión de las aportaciones de la investigación didáctica," Revista Eureka sobre Enseñanza y Divulgación de las Ciencias, Volumen 9, Número 1, 2012, pp. 2-31.

[5] L. Cortés, M. Gómez, J. Calvo, M. Martínez y M. Gil, “Expectativas, necesidades y oportunidades de los maestros en formacion ante la enseñanza de las ciencias en la educación primaria," Enseñanza de las Ciencias, Volumen 3, Número 30, 2012, pp. 155-176.

[6] E. Asencio, “Una alternativa didáctica para el perfeccionamiento del proceso de enseñanza-aprendizaje de las ciencias," Revista Iberoamericana de Educación, Número 58, 2012, pp. 81-97.

[7] G. Moënne, M. Verdi y E. Sepúlveda, "Enseñanza de las ciencias con uso de TIC en escuelas urbano marginales de bajo rendimiento escolar,” en IX Taller Internacional de Software Educativo, Santiago, Chile, 2014.

[8] C. Hernández, “Aproximación a un estado del arte de la enseñanza de las ciencias en Colombia," en La investigación educativa y pedagógica en Colombia, Bogotá, Colciencias - Socolpe, 2011, pp. 1-71.

[9] M. Solarte, “Análisis de contenidos en los textos escolares de ciencias naturales, aplicando la teoría de la transposición didáctica,” EDUCyT, Volumen 1, Número 1, 2010, pp. 175-188.

[10] MinTIC, “Tabletas para Educar,” disponible en http://micrositios.mintic.gov.co/tabletas/que-es.

[11] J. Tovar y G. García, “Investigación en la práctica docente universitaria: obstáculos epistemológicos y alternativas desde la Didáctica General Constructivista,” Educ. Pesqui., Volumen 38, Número 4, 2012, pp. 881-895.

[12] J. Andújar, A. Mejías y M. Márquez, “Augmented Reality for the Improvement of Remote Laboratories: An Augmented Remote Laboratory,” Education, IEEE Transactions on, Volumen 54, Número 3, 2011, pp. $492-500$.

[13] Daqri 4D Studio, “Anatomy 4D,” Disponible en: http://www.4danatomy.com/

[14] Sesame Workshop, “Sesame Street,” Disponible en: http://www.sesamestreet.org/

[15] E. Ascheri, R. Pizarro, G. Astudillo, P. Garcia y M. Culla, "Software educativo en línea para la enseñanza y el aprendizaje de temas de cálculo numérico,” Matemática, Educación e Internet, Volumen 14, Número 2, 2014, pp. 1-28

[16] D. Godoy, E. Belloni, H. Kotynsky, H. Dos Santos y E. Sosa, "Simulando proyectos de desarrollo de software administrado con Scrum," en XVI Workshop de Investigadores en Ciencias de la Computación, Tierra del Fuego, Argentina, 2014.

[17] L. Tovar, J. Bohórquez y P. Puello, “Propuesta metodológica para la construcción de objetos virtuales de aprendizaje basados en realidad aumentada,” Formación Universitaria, Volumen 7, Número 2, 2014, pp. 11-20.

[18] D. Leyton, Extensión al modelo de aceptación de tecnología TAM, para ser aplicado a sistemas colaborativos, en el contexto de pequeñas y medianas empresas. Santiago de Chile, Universidad de Chile, 2013.

[19] GravityJack, “Vuforia Engine,” Disponible en: https://gravityjack.com/vuforia-sdk-gravity-jacks-browsarcode-stack-is-an-augmented-reality-developers-dream 


\section{César Augusto Solano Villanueva}

Nació en Bogotá, Colombia. Es tecnólogo en Sistematización de Datos de la Universidad Distrital Francisco José de Caldas, de Bogotá, Colombia. Actualmente es estudiante de último semestre de Ingeniería Telemática de la Universidad Distrital Francisco José de Caldas, de Bogotá, Colombia. Es líder del semillero de investigación Pegasus e integrante del Grupo de Investigación Metis.

e-mail: casolanov@correo.udistrital.edu.co

\section{Johan Fabiel Casas Díaz}

Nació en Bogotá, Colombia. Es tecnólogo en Sistematización de Datos, de la Universidad Distrital Francisco José de Caldas, de Bogotá, Colombia. Actualmente es estudiante de último semestre de Ingeniería Telemática de la Universidad Distrital Francisco José de Caldas, de Bogotá, Colombia. Además es integrante del semillero de investigación Pegasus y del Grupo de Investigación Metis.

e-mail: Fabie1016@gmail.com

\section{Juan Carlos Guevara Bolaños}

Nació en Bogotá, Colombia. Es Ingeniero de Sistemas de la Universidad Central de Bogotá, Colombia. Obtuvo su título de Maestría en Ciencias de la Información y las Comunicaciones en la Universidad Distrital Francisco José de Caldas, Bogotá, Colombia. Actualmente se desempeña como profesor de tiempo completo en la Universidad Distrital Francisco José de Caldas y es director del Grupo de Investigación Metis.

e-mail: jcguevarab@udistrital.edu.co 